\title{
STABLE IDENTIFICATION OF PIECEWISE-CONSTANT POTENTIALS FROM FIXED-ENERGY PHASE SHIFTS
}

\author{
S. GUTMAN AND ALEXANDER G. RAMM
}

\begin{abstract}
An identification of a spherically symmetric potential by its phase shifts is an important physical problem. Recent theoretical results assure that such a potential is uniquely defined by a sufficiently large subset of its phase shifts at any one fixed energy level. However, two different potentials can produce almost identical phase shifts. That is, the inverse problem of the identification of a potential from its phase shifts at one energy level $k^{2}$ is ill-posed, and the reconstruction is unstable. In this paper we introduce a quantitative measure $D(k)$ of this instability. The diameters of minimizing sets $D(k)$ are used to study the change in the stability with the change of $k$, and the influence of noise on the identification. They are also used in the stopping criterion for the nonlinear minimization method IRRS (Iterative Random Reduced Search). IRRS combines probabilistic global and deterministic local search methods and it is used for the numerical recovery of the potential by the set of its phase shifts. The results of the identification for noiseless as well as noise corrupted data are presented.
\end{abstract}

\section{INTRODUCTION}

Identification of a material from the measurements of its bombardment by particles has long been of interest in physics. The results of such an experiments are used to determine the phase shifts. Identification of a potential by its phase shifts is an important physical problem, see [2, 6, 9, 11, 14]. It has recently been shown that a sufficiently large infinite subset of the set of single energy phase shifts uniquely defines a bounded compactly supported potential, [12]. However, in practice, only a finite (and relatively small) subset of the phase shifts can be determined from experimental data. It has been shown [1, 7, 17, 19 that given such a finite set of shifts at an energy level $k^{2}$ one can find several quite different potentials having practically the same phase shifts at all $l$ (angular momenta) although Ramm's uniqueness theorem 112] guarantees that the phase shifts $\delta_{l}:=\delta(l, k)$, known at a fixed $k>0$ for all values of $l \in \mathcal{L}$, such that $\sum_{l \in \mathcal{L}, l \neq 0} \frac{1}{l}=\infty$, determine uniquely a compactly supported spherically symmetric potential $q(r)$, such that $q(r)=0$ when $r>a>0$ and $\int_{0}^{a} r^{2}|q(r)|^{2} d r<\infty$. Thus, the inverse problem of the recovery of the potential by its phase shifts at one fixed energy level can be severely ill-posed, 15. In this paper we describe a quantitative measure $D(k)$ of such an instability, and an algorithm for its computation. $D(k)$ is the diameter of the minimizing set defined in Section 3. The algorithm provides a method for its numerical estimate. Several numerical examples are presented in Section 4.

Let $q(x), x \in \mathbb{R}^{3}$, be a real-valued potential with compact support. Let $R>0$ be a number such that $q(x)=0$ for $|x|>R$. We also assume that $q \in L^{2}\left(B_{R}\right), B_{R}=\left\{x:|x| \leq R, x \in \mathbb{R}^{3}\right\}$. Let $S^{2}$ be the unit sphere, and $\alpha \in S^{2}$. For a given energy $k>0$ the scattering solution $\psi(x, \alpha)$ is defined as the solution of

$$
\Delta \psi+k^{2} \psi-q(x) \psi=0, \quad x \in \mathbb{R}^{3}
$$

satisfying the following asymptotic condition at infinity:

1991 Mathematics Subject Classification. Primary 35R30, 65K10; Secondary 86A22. 


$$
\begin{gathered}
\psi=\psi_{0}+v, \quad \psi_{0}:=e^{i k \alpha \cdot x}, \quad \alpha \in S^{2}, \\
\lim _{r \rightarrow \infty} \int_{|x|=r}\left|\frac{\partial v}{\partial r}-i k v\right|^{2} d s=0 .
\end{gathered}
$$

It can be shown, that

$$
\psi(x, \alpha)=\psi_{0}+A\left(\alpha^{\prime}, \alpha, k\right) \frac{e^{i k r}}{r}+o\left(\frac{1}{r}\right), \text { as } r \rightarrow \infty, \quad \frac{x}{r}=\alpha^{\prime} .
$$

The function $A\left(\alpha^{\prime}, \alpha, k\right)$ is called the scattering amplitude, $\alpha$ and $\alpha^{\prime}$ are the directions of the incident and scattered waves, and $k^{2}$ is the energy, see [9], [11].

For spherically symmetric scatterers $q(x)=q(r)$ the scattering amplitude satisfies $A\left(\alpha^{\prime}, \alpha, k\right)=$ $A\left(\alpha^{\prime} \cdot \alpha, k\right)$. The converse is established in [13]. Following [18], the scattering amplitude for $q=q(r)$ can be written as

$$
A\left(\alpha^{\prime}, \alpha, k\right)=\sum_{l=0}^{\infty} \sum_{m=-l}^{l} A_{l}(k) Y_{l m}\left(\alpha^{\prime}\right) \overline{Y_{l m}(\alpha)}
$$

where $Y_{l m}$ are the spherical harmonics, normalized in $L^{2}\left(S^{2}\right)$, and the bar denotes the complex conjugate.

The fixed-energy phase shifts $-\pi<\delta_{l} \leq \pi\left(\delta_{l}=\delta(l, k), k>0\right.$ is fixed) are related to $A_{l}(k)$ (see e.g., 18) by the formula:

$$
A_{l}(k)=\frac{4 \pi}{k} e^{i \delta_{l}} \sin \left(\delta_{l}\right) .
$$

\section{Phase Shifts for Piecewise-Constant Potentials}

In general, phase shifts for a spherically symmetric potentials can be computed by a variety of methods, e.g. by a variable phase method described in [4]. The computation involves solving a nonlinear ODE for each phase shift. However, if the potential is compactly supported and piecewise-continuous, a much simpler method described in [1] can be used. It is summarized below.

Consider a finite set of points $0=r_{0}<r_{1}<r_{2}<\cdots<r_{N}=R$ and a piecewise-constant potential

$$
q(r)=q_{i}, \text { on }\left[r_{i-1}, r_{i}\right) \text { for } i=1, \ldots, N \text {, and } q=0 \text { for } r \geq R .
$$

Denote $\kappa_{i}^{2}:=k^{2}-q_{i}$, where $i=1, \ldots, N$, and $k$ is some fixed positive number. Consider the following problem for the radial Schrödinger equation:

$$
\frac{d^{2} \varphi_{l}}{d r^{2}}+\left(k^{2}-\frac{l(l+1)}{r^{2}}\right) \varphi_{l}=q \varphi_{l}, \quad \lim _{r \rightarrow 0}(2 l+1) ! ! r^{-l-1} \varphi_{l}(r)=1,
$$

which we rewrite as:

$$
\frac{d^{2} \varphi_{l}}{d r^{2}}+\left(\kappa_{i}^{2}-\frac{l(l+1)}{r^{2}}\right) \varphi_{l}=0
$$

on the interval $r_{i-1} \leq r<r_{i}$. On $\left[r_{i-1}, r_{i}\right)$ one has the following general solution of (2.3) 


$$
\varphi_{l}(r)=A_{i} j_{l}\left(\kappa_{i} r\right)+B_{i} n_{l}\left(\kappa_{i} r\right),
$$

where

$$
j_{l}(k r)=\sqrt{\frac{\pi k r}{2}} J_{l+1 / 2}(k r), n_{l}(k r)=\sqrt{\frac{\pi k r}{2}} N_{l+1 / 2}(k r)
$$

and $J_{l}, N_{l}$ are the Bessel and Neumann functions.

We assume below that $\kappa_{i}$ does not vanish for all $i$. If $\kappa_{i}=0$ for some $i$, then (2.3) has the solution

$$
\varphi_{l}(r)=A_{i} r^{l+1}+B_{i} r^{-l},
$$

and our approach is still valid with obvious changes.

From the regularity of $\varphi_{l}$ at zero one gets $B_{1}=0$. Denote $x_{i}=B_{i} / A_{i}$, then $x_{1}=0$. We are looking for the continuously differentiable solution $\varphi_{l}$. Thus, the following interface conditions hold:

$$
\begin{aligned}
& A_{i} j_{l}\left(\kappa_{i} r_{i}\right)+B_{i} n_{l}\left(\kappa_{i} r_{i}\right)=A_{i+1} j_{l}\left(\kappa_{i+1} r_{i}\right)+B_{i+1} n_{l}\left(\kappa_{i+1} r_{i}\right), \\
& \frac{\kappa_{i}}{\kappa_{i+1}}\left[A_{i} j_{l}^{\prime}\left(\kappa_{i} r_{i}\right)+B_{i} n_{l}^{\prime}\left(\kappa_{i} r_{i}\right)\right]=A_{i+1} j_{l}^{\prime}\left(\kappa_{i+1} r_{i}\right)+B_{i+1} n_{l}^{\prime}\left(\kappa_{i+1} r_{i}\right) .
\end{aligned}
$$

The Wronskian $W\left(j_{l}(r), n_{l}(r)\right)=1$, thus

$$
\begin{aligned}
& A_{i+1}=n_{l}^{\prime}\left(\kappa_{i+1} r_{i}\right)\left[A_{i} j_{l}\left(\kappa_{i} r_{i}\right)+B_{i} n_{l}\left(\kappa_{i} r_{i}\right)\right]-\frac{\kappa_{i}}{\kappa_{i+1}} n_{l}\left(\kappa_{i+1} r_{i}\right)\left[A_{i} j_{l}^{\prime}\left(\kappa_{i} r_{i}\right)+B_{i} n_{l}^{\prime}\left(\kappa_{i} r_{i}\right)\right], \\
& B_{i+1}=\frac{\kappa_{i}}{\kappa_{i+1}} j_{l}\left(\kappa_{i+1} r_{i}\right)\left[A_{i} j_{l}^{\prime}\left(\kappa_{i} r_{i}\right)+B_{i} n_{l}^{\prime}\left(\kappa_{i} r_{i}\right)\right]-j_{l}^{\prime}\left(\kappa_{i+1} r_{i}\right)\left[A_{i} j_{l}\left(\kappa_{i} r_{i}\right)+B_{i} n_{l}\left(\kappa_{i} r_{i}\right)\right] .
\end{aligned}
$$

Therefore

$$
\left(\begin{array}{l}
A_{i+1} \\
B_{i+1}
\end{array}\right)=\frac{1}{\kappa_{i+1}}\left(\begin{array}{ll}
\alpha_{11}^{i} & \alpha_{12}^{i} \\
\alpha_{21}^{i} & \alpha_{22}^{i}
\end{array}\right)\left(\begin{array}{c}
A_{i} \\
B_{i}
\end{array}\right)
$$

where the entries of the matrix $\alpha^{i}$ can be written explicitly:

$$
\begin{aligned}
& \alpha_{11}^{i}=\kappa_{i+1} j_{l}\left(\kappa_{i} r_{i}\right) n_{l}^{\prime}\left(\kappa_{i+1} r_{i}\right)-\kappa_{i} j_{l}^{\prime}\left(\kappa_{i} r_{i}\right) n_{l}\left(\kappa_{i+1} r_{i}\right), \\
& \alpha_{12}^{i}=\kappa_{i+1} n_{l}\left(\kappa_{i} r_{i}\right) n_{l}^{\prime}\left(\kappa_{i+1} r_{i}\right)-\kappa_{i} n_{l}^{\prime}\left(\kappa_{i} r_{i}\right) n_{l}\left(\kappa_{i+1} r_{i}\right), \\
& \alpha_{21}^{i}=\kappa_{i} j_{l}^{\prime}\left(\kappa_{i} r_{i}\right) j_{l}\left(\kappa_{i+1} r_{i}\right)-\kappa_{i+1} j_{l}\left(\kappa_{i} r_{i}\right) j_{l}^{\prime}\left(\kappa_{i+1} r_{i}\right), \\
& \alpha_{22}^{i}=\kappa_{i} n_{l}^{\prime}\left(\kappa_{i} r_{i}\right) j_{l}\left(\kappa_{i+1} r_{i}\right)-\kappa_{i+1} n_{l}\left(\kappa_{i} r_{i}\right) j_{l}^{\prime}\left(\kappa_{i+1} r_{i}\right) .
\end{aligned}
$$

Thus

$$
x_{i+1}=\frac{\alpha_{21}^{i}+\alpha_{22}^{i} x_{i}}{\alpha_{11}^{i}+\alpha_{12}^{i} x_{i}}, \quad x_{i}:=\frac{B_{i}}{A_{i}}
$$

The phase shift $\delta(k, l)$ is defined by

$$
\varphi_{l}(r) \sim \frac{|F(k, l)|}{k^{l+1}} \sin \left(k r-\frac{\pi l}{2}+\delta(k, l)\right) \quad r \rightarrow \infty,
$$

where $F(k, l)$ is the Jost function. For $r>R$

$$
\varphi_{l}(r)=A_{N+1} j_{l}(k r)+B_{N+1} n_{l}(k r) .
$$


From (2.13) and the asymptotics $j_{l}(k r) \sim \sin (k r-l \pi / 2), \quad n_{l}(k r) \sim-\cos (k r-l \pi / 2), r \rightarrow \infty$, one gets:

$$
\tan \delta(k, l)=-\frac{B_{N+1}}{A_{N+1}}=-x_{N+1}
$$

Finally, the phase shifts of the potential $q(r)$ are calculated by the formula:

$$
\delta(k, l)=-\arctan x_{N+1} .
$$

Let $q_{0}(r)$ be a spherically symmetric piecewise-constant potential. Fix an energy level $k$ and a sufficiently large $N$. Let $\{\tilde{\delta}(k, l)\}_{l=1}^{N}$ be the set of its phase shifts. Let $q(r)$ be another such potential, and let $\{\delta(k, l)\}_{l=1}^{N}$ be the set of its phase shifts.

The best fit to data function $\Phi(q, k)$ is defined by

$$
\Phi(q, k)=\frac{\sum_{l=1}^{N}|\delta(k, l)-\tilde{\delta}(k, l)|^{2}}{\sum_{l=1}^{N}|\tilde{\delta}(k, l)|^{2}},
$$

The phase shifts are known to decay rapidly with $l$, see [16]. Thus, for sufficiently large $N$, the function $\Phi$ is practically the same as the one which would use all the shifts in (2.16). The inverse problem of the reconstruction of the potential from its fixed-energy phase shifts is reduced to the minimization of the objective function $\Phi$ over an appropriate admissible set. A minimization algorithm for this nonlinear problem providing a stability estimate for the identification of the original potential $q_{0}$ is given in the next Section.

\section{Global and Local Minimization Methods}

We seek the potentials $q(r)$ in the class of piecewise-constant, spherically symmetric real-valued functions. Let the admissible set be

$$
A_{a d m} \subset\left\{\left(r_{1}, r_{2}, \ldots, r_{M}, q_{1}, q_{2}, \ldots, q_{M}\right): 0 \leq r_{i} \leq R, q_{\text {low }} \leq q_{m} \leq q_{\text {high }}\right\},
$$

where the bounds $q_{\text {low }}$ and $q_{\text {high }}$ for the potentials, as well as the bound $M$ on the expected number of layers are assumed to be known.

A configuration $\left(r_{1}, r_{2}, \ldots, r_{M}, q_{1}, q_{2}, \ldots, q_{M}\right)$ corresponds to the potential

$$
q(r)=q_{m}, \quad \text { for } \quad r_{m-1} \leq r<r_{m}, \quad 1 \leq m \leq M,
$$

where $r_{0}=0$ and $q(r)=0$ for $r \geq r_{M}=R$.

Note, that the admissible configurations must also satisfy

$$
r_{1} \leq r_{2} \leq r_{3} \leq \cdots \leq r_{M}
$$

Given an initial configuration $Q_{0} \in A_{a d m} \subset \mathbb{R}^{2 M}$, a local minimization method finds a local minimum near $Q_{0}$. On the other hand, global minimization methods explore the entire admissible set in order to find a global minimum of the objective function. While the local minimization is usually deterministic, the majority of the global methods are probabilistic. As usual for inverse scattering problems, the best fit to data function $\Phi$ has many local minima and points of nondifferentiability, see [7]. In this situation a combination of global probabilistic and local deterministic methods proved to be successful.

In 8 such an algorithm (the Hybrid Stochastic-Deterministic Method) has been applied for the identification of small subsurface particles, given a set of surface measurements. The HSD method could be described as a variation of a genetic algorithm and a local search with reduction. In [6] 
two global search algorithms in combination with a special local search method were applied to the identification of piecewise-constant scatterers by acoustic type measurements. The Rinnooy Kan and Timmer's Multilevel Single-Linkage Method in a combination with a special Local Minimization Method has been applied to the identification of piecewise-constant spherically symmetric potentials by their phase shifts in [7]. We have used the Reduced Random Search Method in [17] to find different potentials with practically the same phase shifts.

In this paper we use the Modified Reduced Random Search Method. The important modification consists of the consideration of minimizing sets and their diameters as quantitative measures of the stability of the minimization algorithm.

In a pure Random Search method a batch $H$ of $L$ trial points is generated in $A_{a d m}$ using a uniformly distributed random variable. Then a local search is started from each of these $L$ points. A local minimum with the smallest value of $\Phi$ is declared to be the global one.

In our case $A_{a d m}$ is a box in $\mathbb{R}^{2 M}$. The uniform random variable is called $2 M$ times to produce a point in this box (after the appropriate rescaling in each dimension). Finally, the obtained values of $r_{i}$ are rearranged in the ascending order to satisfy (3.3).

Since the Random Search method is computationally extremely inefficient, it is modified to reduce the number of local searches. In the Reduced Sample Random Search method one uses only a certain fixed fraction $\gamma$ of the original batch of $L$ points to proceed with the local searches. Typically, $L=5000$ and $\gamma=0.01$. This reduced sample $H_{\text {red }}$ of $\gamma L$ points is chosen to contain the points with the smallest $\gamma L$ values of $\Phi$ among the original batch. The local searches are started from the points in this reduced sample. This way only the points that seem to be in a neighborhood of the global minimum are used for an expensive local minimization, and the computational time is not wasted on less promising candidates.

Let $H_{\min }$ be the $\gamma L$ points obtained as the result of the local minimizations ( $\gamma L=50$ in our computations). Let $S_{\min }$ be the subset of $H_{\min }$ containing points $\left\{p_{i}\right\}$ with the smallest $\nu \gamma L$ $(0<\nu<1$, we used $\nu=0.1)$ values in $H_{\min }$. We call $S_{\min }$ the minimizing set. The choice of $\nu$ determines a representative sample of global minimizers. If all these minimizers are close to each other, then the objective function $\Phi$ is not flat near the global minimum. That is, the method identifies the minimum consistently. To define this consistency in quantitative terms, let $\|\cdot\|$ be a norm in the admissible set.

Let

$$
D=\operatorname{diam}\left(S_{m i n}\right)=\max \left\{\left\|p_{i}-p_{j}\right\| / d_{a v}: p_{i}, p_{j} \in S_{m i n}\right\},
$$

where $d_{a v}$ is the average norm of the elements in $H_{\text {min }}$. The normalization by $d_{a v}$ is introduced to provide comparable results for different potentials.

Large $D$ indicates that the found minimizers $p_{i}$ of $\Phi$ are far apart. In terms of the Inverse Problem, it means that the found solution is not stable. The diameter $D$ is a measure of such instability. A detailed description of an iterative version of this algorithm is given at the end of this Section. First, we discuss local minimization methods.

In our minimization algorithm the Reduced Sample Random Search method is coupled with a deterministic Local Minimization Method. Numerical experiments show that the objective function $\Phi$ is relatively well behaved in this problem: while it contains many local minima and, at some points, $\Phi$ is not differentiable, standard minimization methods work well here. A Newton-type method for the minimization of $\Phi$ is described in [1. We have chosen to use a variation of Powell's minimization method which does not require the computation of the derivatives of the objective function. Such method needs a minimization routine for a one-dimensional minimization of $\Phi$, which we do using a Bisection or a Golden Rule method. See [6] or [7] for a complete description of our method. 
Now we can describe our Basic Local Minimization Method in $\mathbb{R}^{2 M}$, which is a modification of Powell's minimization method [3]. It is assumed here that the starting position (configuration) $Q_{0} \in A_{a d m}$ is suppied by the procedure LMM (see below), and the entry to LMM is provided by the global minimization part (IRRS).

\section{Basic Local Minimization Method.}

1. Choose the set of directions $u_{i}, i=1,2, \ldots, 2 M$ to be the standard basis in $\mathbb{R}^{2 M}$

$$
u_{i}=(0,0, \ldots, 1, \ldots, 0) \text {, }
$$

where 1 is in the i-th place.

2. Save your starting configuration supplied by LMM as $Q_{0}$.

3. For each $i=1, \ldots, 2 M$ move from $Q_{0}$ along the line defined by $u_{i}$ and find the point of minimum $Q_{i}^{t}$. This defines $2 M$ temporary points of minima.

4. Re-index the directions $u_{i}$, so that (for the new indices) $\Phi\left(Q_{1}^{t}\right) \leq \Phi\left(Q_{2}^{t}\right) \leq, \ldots, \Phi\left(Q_{2 M}^{t}\right) \leq$ $\Phi\left(Q_{0}\right)$.

5. For $i=1, \ldots, 2 M$ move from $Q_{i-1}$ along the direction $u_{i}$ and find the point of minimum $Q_{i}$.

6. Set $v=Q_{2 M}-Q_{0}$.

7. Move from $Q_{0}$ along the direction $v$ and find the minimum. Call it $Q_{0}$ again. It replaces $Q_{0}$ from step 2.

8. Repeat the above steps until a stopping criterion is satisfied.

Note, that we use the temporary points of minima $Q_{i}^{t}$ only to rearrange the initial directions $u_{i}$ in a different order. The stopping criterion is the same as the one in [10, Subroutine Powell].

Still another refinement of the local phase is necessary to produce a successful minimization. The admissible set $A_{\text {adm }}$, see (3.1)-(3.3), belongs to a $2 M$ dimensional minimization space $\mathbb{R}^{2 M}$. The dimension $2 M$ of this space is chosen a priori to be larger than $2 N$, where $N$ is the number of layers in the original potential. We have chosen $M=6$ in our numerical experiments. However, since the sought potential may have fewer than $M$ layers, we found that conducting searches in lower-dimensional subspaces of $\mathbb{R}^{2 M}$ is essential for the local minimization phase. A variation of the following "reduction" procedure has also been found to be necessary in [8] for the search of small subsurface objects, and in $[6]$ for the identification of multilayered scatterers.

If two adjacent layers in a potential have values $v_{i-1}$ and $v_{i}$ and the objective function $\Phi$ is not changed much when both layers are assigned the same value $v_{i}$ (or $v_{i-1}$ ), then these two layers can be replaced with just one layer occupying their place. The change in $\Phi$ is controlled by the parameter $\epsilon_{r}$. We used $\epsilon_{r}=0.1$. This value, found from numerical experiments, seems to provide the most consistent identification. The minimization problem becomes constrained to a lower dimensional subspace of $\mathbb{R}^{2 M}$ and the local minimization is done in this subspace.

Reduction Procedure. Let $\epsilon_{r}$ be a positive number.

1. Save your starting configuration $Q_{0}=\left(r_{1}, r_{2}, \ldots, r_{M}, v_{1}, v_{2}, \ldots, v_{M}\right) \in A_{a d m}$ and the value $\Phi\left(Q_{0}\right)$. Let the $(M+1)$-st layer be $L_{M+1}=\left\{r_{M} \leq|x| \leq R\right\}$ and $v_{M+1}=0$.

2. Let $2 \leq i \leq M+1$. Replace $v_{i-1}$ in the layer $L_{i-1}$ by $v_{i}$. This defines a new configuration $Q_{i}^{d}$, where the layers $L_{i-1}$ and $L_{i}$ are replaced with one new layer. Here $d$ stands for the downward adjustment. Compute $\Phi\left(Q_{i}^{d}\right)$ and the difference $c_{i}^{d}=\left|\Phi\left(Q_{0}\right)-\Phi\left(Q_{i}^{d}\right)\right|$. Repeat for each layer in the original configuration $Q_{0}$.

3. Let $1 \leq i \leq M$. Replace $v_{i+1}$ in the layer $L_{i+1}$ by $v_{i}$. This defines a new configuration $Q_{i}^{u}$, where the layers $L_{i}$ and $L_{i+1}$ are replaced with one new layer. Here $u$ stands for the upward adjustment. Compute $\Phi\left(Q_{i}^{u}\right)$ and the difference $c_{i}^{u}=\left|\Phi\left(Q_{0}\right)-\Phi\left(Q_{i}^{u}\right)\right|$. Repeat for each layer in the original configuration $Q_{0}$. 
4. Find the smallest among the numbers $c_{i}^{d}$ and $c_{i}^{u}$. If this number is less than $\epsilon_{r} \Phi\left(Q_{0}\right)$, then implement the adjustment that produced this number. The resulting new configuration has one less layer than the original configuration $Q_{0}$.

5. Repeat the above steps until no further reduction in the number of layers is occurring.

Note, that an application of the Reduction Procedure may or may not result in the actual reduction of the number of layers.

Finally, the entire Local Minimization Method (LMM) consists of the following:

\section{Local Minimization Method (LMM).}

1. Let your starting configuration supplied by IRRS be $Q_{0}=\left(r_{1}, r_{2}, \ldots, r_{M}, v_{1}, v_{2}, \ldots, v_{M}\right) \in$ $A_{\text {adm }}$.

2. Apply the Reduction Procedure to $Q_{0}$, and obtain a reduced configuration $Q_{0}^{r}$ containing $M^{r}$ layers.

3. Apply the Basic Minimization Method in $A_{a d m} \cap \mathbb{R}^{2 M^{r}}$ with the starting configuration $Q_{0}^{r}$, and obtain a configuration $Q_{1}$.

4. Apply the Reduction Procedure to $Q_{1}$, and obtain a final reduced configuration $Q_{1}^{r}$.

As we have already mentioned, LMM is used as the local phase of the global minimization. The global part is described as follows:

Iterative Reduced Random Search (IRRS). (at the $j$-th iteration).

Fix $0<\gamma, \nu, \beta<1, \epsilon>0$ and $j_{\max }$.

1. Generate another batch $H^{j}$ of $L$ trial points (configurations) in $A_{a d m}$ using a uniform random distribution.

2. Reduce $H^{j}$ to the reduced sample $H_{\text {red }}^{j}$ of $\gamma L$ points by selecting the points in $H^{j}$ with the smallest $\gamma L$ values of $\Phi$.

3. Apply the Local Minimization Method (LMM) starting it at each point in $H_{\text {red }}^{j}$, and obtain the set $H_{m i n}^{j}$ consisting of the $\gamma L$ minimizers.

4. Combine $H_{\min }^{j}$ with $H_{\min }^{j-1}$ obtained at the previous iteration. Let $S_{\min }^{j}$ be the set of points from $H_{\min }^{j} \cup H_{\min }^{j-1}$ with the smallest $\nu \gamma L$ values of $\Phi$. (Use $H_{\min }^{1}$ for $j=1$ ).

5. Compute the diameter $D^{j}$ of $S_{\text {min }}^{j}$ by $D^{j}=\max \left\{\left\|p_{i}-p_{k}\right\| / d_{a v}: p_{i}, p_{k} \in S_{\min }\right\}$, where $d_{a v}$ is the average norm of the $\gamma L$ elements of $H_{\text {min }}^{j} \cup H_{m i n}^{j-1}$ with the smallest values of $\Phi$.

6. (Stopping criterion).

Let $p \in S_{\text {min }}^{j}$ be the point with the smallest value of $\Phi$ in $S_{\min }^{j}$ (the global minimizer).

If $D^{j} \leq \epsilon$, then stop. The global minimum is $p$. The minimization is stable.

If $D^{j}>\epsilon$ and $D^{j} \leq \beta D^{j-1}$, then return to step 1, and do another iteration. (Stop, if the maximum number of iterations $j_{\max }$ is exceeded).

If $D^{j}>\epsilon$ and $D^{j}>\beta D^{j-1}$, then stop. $p$ is the global minimum. The minimization is unstable. The diameter $D^{j}$ is the measure of the instability of the minimization.

We used $\beta=0.95, \epsilon=0.01$ and $j_{\max }=6$. The choice of these and other parameters $(L=$ $\left.5000, \gamma=0.01, \nu=0.1 \epsilon_{r}=0.1\right)$ is dictated by their meaning in the algorithm and the comparative performance of the program at their different values. As usual, some adjustment of parameters, stopping criteria etc. is needed to achieve an optimal performance of the algorithm.

\section{Numerical Results}

We studied the performance of the algorithm for 4 different potentials $q_{i}(r), i=1,2,3,4$. In each case the following values of the parameters have been used. The radius $R$ of the support of each $q_{i}$ was chosen to be $R=3.0$. The admissible set $A_{a d m}$ (3.1) was defined with $M=8$. The Reduced Random Search parameters: $L=5000, \gamma=0.01, \nu=0.1, \epsilon=0.01, \beta=0.95, j_{\max }=6$. The 
TABLE 1. Phase shifts of $q_{1}(r)$ for $k=9$.

\begin{tabular}{rr|rr|rr}
\hline$l$ & $\tilde{\delta}(k, l)$ & $l$ & $\tilde{\delta}(k, l)$ & $l$ & $\tilde{\delta}(k, l)$ \\
\hline 0 & $-0.95151654 \mathrm{D}-01$ & 12 & $0.43249567 \mathrm{D}-01$ & 24 & $-0.58868576 \mathrm{D}-03$ \\
1 & $-0.59487863 \mathrm{D}-01$ & 13 & $0.78575610 \mathrm{D}-01$ & 25 & $-0.15074621 \mathrm{D}-03$ \\
2 & $-0.30344479 \mathrm{D}-01$ & 14 & $0.27082102 \mathrm{D}-01$ & 26 & $-0.34641742 \mathrm{D}-04$ \\
3 & $-0.36224576 \mathrm{D}-01$ & 15 & $-0.10281029 \mathrm{D}+00$ & 27 & $-0.71940777 \mathrm{D}-05$ \\
4 & $0.14419664 \mathrm{D}-01$ & 16 & $-0.18261448 \mathrm{D}+00$ & 28 & $-0.13582152 \mathrm{D}-05$ \\
5 & $-0.35167060 \mathrm{D}-01$ & 17 & $-0.17579851 \mathrm{D}+00$ & 29 & $-0.23433795 \mathrm{D}-06$ \\
6 & $0.38359584 \mathrm{D}-02$ & 18 & $-0.12758628 \mathrm{D}+00$ & 30 & $-0.37119174 \mathrm{D}-07$ \\
7 & $0.40280065 \mathrm{D}-01$ & 19 & $-0.76312741 \mathrm{D}-01$ & 31 & $-0.54203180 \mathrm{D}-08$ \\
8 & $0.45775379 \mathrm{D}-01$ & 20 & $-0.38650348 \mathrm{D}-01$ & 32 & $-0.73237693 \mathrm{D}-09$ \\
9 & $0.99311592 \mathrm{D}-01$ & 21 & $-0.16752224 \mathrm{D}-01$ & & \\
10 & $0.93668476 \mathrm{D}-01$ & 22 & $-0.62688318 \mathrm{D}-02$ & & \\
11 & $0.32078999 \mathrm{D}-01$ & 23 & $-0.20460976 \mathrm{D}-02$ & & \\
\hline \multicolumn{7}{|c}{}
\end{tabular}

value $\epsilon_{r}=0.1$ was used in the Reduction Procedure (see Section 3) during the local minimization phase. The initial configurations were generated using a random number generator with seeds determined by the system time. The run time was between 30 minutes to 2 hours on a $333 \mathrm{MHz}$ $\mathrm{PC}$, depending on the wave number $k$. The number $N$ of the shifts used in (2.16) for the formation of the objective function $\Phi(q)$ was determined from the condition $\delta(k, 0)>10^{-7} \delta(k, l)$. So, it was different for different potentials $q_{i}$ and different wave numbers $k$. The upper and lower bounds for the potentials $q_{l o w}$ and $q_{h i g h}$ used in the definition of the admissible set $A_{a d m}$ were chosen to reflect the presumed a priori information about the potentials. While one may attempt to deduce the bounds from the set of given data, i.e. the phase shifts, it turns out, that an extensive admissible set may lead to a nonuniqueness in the sought potential. This issue will be studied elsewhere.

Let $q_{1}(r)$ be the following potential

$$
q_{1}(r)= \begin{cases}4.0 & 0 \leq r<0.3 \\ 1.0 & 0.3 \leq r<1.0 \\ -2.0 & 1.0 \leq r<1.9 \\ 3.5 & 1.9 \leq r<2.2 \\ 1.0 & 2.2 \leq r<2.4 \\ 0.0 & r \geq 2.4\end{cases}
$$

This potential is shown in Figure 1. The upper and lower bounds for the potential $q_{\text {low }}=-5.0$ and $q_{\text {high }}=5.0$ were used for all the wave numbers $k=3,4,5,6,7,8,9$.

The phase shifts $\tilde{\delta}(k, l)$ for $k=9$ (computed as in Section 2) are shown in Table 1 .

The identification was attempted with 3 different noise levels $h$. The levels are $h=0.0$ (no noise), $h=0.0001$ and $h=0.001$. More precisely, the noisy phase shifts $\delta_{h}(k, l)$ were obtained from the exact phase shifts $\delta(k, l)$ by the formula

$$
\delta_{h}(k, l)=\delta(k, l)+(0.5-z) \cdot h \cdot \delta_{\max },
$$

where $\delta_{\max }=\max \left\{\left|\delta_{h}(k, l)\right|: l=0,1, \ldots, N\right\}$, and $z$ is the uniformly distributed on $[0,1]$ random variable.

The distance $d\left(p^{1}(r), p^{2}(r)\right)$ for any two potentials in step 5 of the IRRS algorithm was computed as 
TABLE 2. Diameters $D$ of minimizing sets for $q_{1}(r)$ at different noise levels $h$.

\begin{tabular}{rrrr}
\hline$k$ & $h=0.000$ & $h=0.0001$ & $h=0.001$ \\
\hline 3 & 0.886140 & 1.062616 & 0.870692 \\
4 & 0.653900 & 0.629565 & 1.017525 \\
5 & 0.636675 & 0.661651 & 0.754354 \\
6 & 0.456157 & 0.326852 & 0.585782 \\
7 & 0.008116 & 0.011184 & 0.066413 \\
8 & 0.014142 & 0.004978 & 0.010448 \\
9 & 0.007881 & 0.011849 & 0.022112 \\
\hline
\end{tabular}

TABle 3. Diameters $D$ of minimizing sets for $q_{2}(r)$ at different noise levels $h$.

\begin{tabular}{rrrr}
\hline$k$ & $h=0.000$ & $h=0.0001$ & $h=0.001$ \\
\hline 3 & 1.184754 & 1.865304 & 1.094802 \\
4 & 1.435954 & 0.843646 & 0.887367 \\
5 & 0.700282 & 0.684679 & 1.032036 \\
6 & 0.000568 & 0.000990 & 0.693338 \\
7 & 0.000373 & 0.001486 & 0.420729 \\
8 & 0.000294 & 0.001435 & 0.003349 \\
9 & 0.000190 & 0.001218 & 0.005308 \\
\hline
\end{tabular}

$$
d\left(p^{1}(r), p^{2}(r)\right)=\left\|p^{1}(r)-p^{2}(r)\right\|
$$

where the norm is the $L_{2}$-norm in $\mathbb{R}^{3}$.

The results of the identification algorithm (the diameters of the minimizing sets as the function of the wave number $k$ ) for the potential $q_{1}(r)$ are shown in Table 2 as well as in Figure 2.

The diameter $D \leq 0.01$ indicates that the potentials in the minimizing set are, practically, undistinguishable. That is, the identification is stable for $k \geq 7$ and a low noise level.

The second potential $q_{2}(r)$ is defined by

$$
q_{2}(r)= \begin{cases}2.0 & 0 \leq r<0.5 \\ 1.0 & 0.5 \leq r<1.0 \\ 2.0 & 1.0 \leq r<1.5 \\ 1.0 & 1.5 \leq r<2.0 \\ 0.0 & r \geq 2.0\end{cases}
$$

This positive 4-layer potential is shown in Figure 3. The upper and lower bounds for the admissible set were the same as for $q_{1}$. The results of the identification are shown in Table 3 and in Figure 4. The identification is stable for $k \geq 6$ for low noise levels. It is becoming stable for the noise level $h=0.001$ at $k=8$.

We have also considered the identification for small potentials. In this case the potential has to be identified from relatively small phase shifts. We defined $q_{3}(r)=0.1 q_{2}(r)$, and $q_{4}(r)=0.01 q_{2}(r)$. It turns out, that the identification is comparable in quality to the ones above, provided that reasonable a priori bounds for the potentials are supplied. We used $q_{\text {low }}=-0.5$ and $q_{\text {high }}=0.5$ for the identification of potential $q_{3}(r)$, and $q_{l o w}=-0.05$ and $q_{\text {high }}=0.05$ for potential $q_{4}(r)$. The results of the identification are shown in Tables 4 and 5, as well as in Figures 5 and 6. 
TABLE 4 . Diameters $D$ of minimizing sets for $q_{3}=0.1 q_{2}$ at different noise levels $h$.

\begin{tabular}{rrrr}
\hline$k$ & $h=0.000$ & $h=0.0001$ & $h=0.001$ \\
\hline 3 & 0.914191 & 0.958988 & 1.271533 \\
4 & 0.649307 & 1.432330 & 0.571411 \\
5 & 0.258550 & 0.425632 & 0.599525 \\
6 & 0.000541 & 0.001361 & 0.318754 \\
7 & 0.000373 & 0.211909 & 0.004215 \\
8 & 0.000295 & 0.002696 & 0.006563 \\
9 & 0.000170 & 0.003122 & 0.007665 \\
\hline
\end{tabular}

TABle 5. Diameters $D$ of minimizing sets for $q_{4}=0.01 q_{2}$ at different noise levels $h$.

\begin{tabular}{rrrr}
\hline$k$ & $h=0.000$ & $h=0.0001$ & $h=0.001$ \\
\hline 3 & 1.233750 & 0.882006 & 1.681609 \\
4 & 1.249050 & 0.891463 & 0.683901 \\
5 & 0.676837 & 1.082434 & 0.309618 \\
6 & 0.000565 & 0.001337 & 0.136704 \\
7 & 0.000270 & 0.001288 & 0.004241 \\
8 & 0.000523 & 0.001006 & 0.002867 \\
9 & 0.000358 & 0.000991 & 0.005288 \\
\hline
\end{tabular}

\section{Conclusions}

Recovery of a spherically symmetric potential from its fixed-energy phase shifts is a classical physical problem. Recent theoretical results [12 assure that such a potential is uniquely defined by a sufficiently large subset of its phase shifts at any one fixed energy level. However, two different potentials can produce almost identical fixed-energy phase shifts ([1], [17]). That is, the inverse problem of the identification of the potential by its fixed-energy phase shifts is very unstable. In this paper we investigate the instability of the inversion by an Iterative Reduced Random Search Method (IRRS). The diameter of the minimizing set $D$ is introduced to provide a quantitative measure for the instability. It also serves as the stopping criterion in the IRRS algorithm. The results show, that for several types of piecewise-constant spherically symmetric potentials the identification is becoming stable $(D \leq 0.01)$ for phase shifts measured at a higher energy level. It is also shown that the introduction of a low noise level into the data does not significantly degrades the identification. This method can serve as a tool for experimentalists to determine if a particular set of phase shifts would produce a stable identification of the underlying potential, or a higher energy level should be used.

\section{REFERENCES}

[1] Airapetyan R., Ramm A.G. and Smirnova A.B. [1999] Example of two different potentials which have practical the same fixed-energy phase shifts, Phys. Lett A, 254, 141-148.

[2] Apagyi, B, et al (Eds) [1997] Inverse and algebraic quantum scattering theory, Springer, Berlin.

[3] Brent P. [1973] Algorithms for minimization without derivatives, Prentice-Hall, Englewood Cliffs, NJ.

[4] Calogero F. [1967] Variable Phase Approach to Potential Scattering, Academic Press, New York and London.

[5] Chadan K., Sabatier P. [1989] Inverse Problems in Quantum Scattering Theory, Springer Verlag.

[6] Gutman S. [2000] Identification of multilayered particles from scattering data by a clustering method, J. Comp. Phys., 163, 529-546.

[7] Gutman S. Identification of piecewise-constant potentials by fixed-energy shifts, submitted.

[8] Gutman S. and Ramm A.G. [2000] Application of the Hybrid Stochastic-deterministic Minimization Method to a Surface Data Inverse Scattering Problem, Fields Institute Communications, 25, 293-304. 
[9] Newton R. [1982] Scattering Theory of Waves and Particles, Springer Verlag.

[10] Press W.H., Teukolsky S.A., Vetterling W.T., Flannery B.P. [1992] Numerical Recepies in FORTRAN, Second Ed., Cambridge University Press.

[11] Ramm A.G. [1992] Multidimensional inverse scattering problems, Longman/Wiley, New York, Expanded Russian edition, MIR, Moscow, 1994.

[12] Ramm A.G. [1999] An inverse scattering problem with part of the fixed-energy phase shifts, Comm. Math. Phys., 207, N1, 231-247.

[13] Ramm A.G. [1991] Symmetry properties for scattering amplitudes and applications to inverse problems, J. Math. Anal. Appl., 156, 333-340.

[14] Ramm A.G. [1988] Recovery of the potential from fixed-energy scattering data, Inverse Problems, 4, 877-886.

[15] Ramm A.G. [1992] Stability estimates in inverse scattering, Acta Appl. Math., 28, N1, 1-42.

[16] Ramm A.G., Arredondo J.H., Izquierdo B.G. [1998] Formula for the radius of the support of the potential in terms of the scattering data, Jour. Phys. A, 31, N1, L39-L44.

[17] Ramm A.G. and Gutman S. Piecewise-constant positive potentials with practically the same fixed-energy phase shifts, submitted.

[18] Ramm A.G. and Scheid W. [1999] An approximate method for solving inverse scattering problem with fixedenergy data Jour. of Inverse and Ill-Posed Problems, 7, N6, 561-571.

[19] Ramm A.G. and Smirnova A. [2000] A numerical method for solving the inverse scattering problem with fixed-energy phase shifts, Jour. of Inverse and Ill-Posed Problems, 8, N3, 307-322.

Department of Mathematics, University of Oklahoma, Norman, OK 73019, USA

E-mail address: sgutman@ou.edu

Department of Mathematics, Kansas State University, Manhattan, Kansas 66506-2602, USA

E-mail address: ramm@math.ksu.edu 


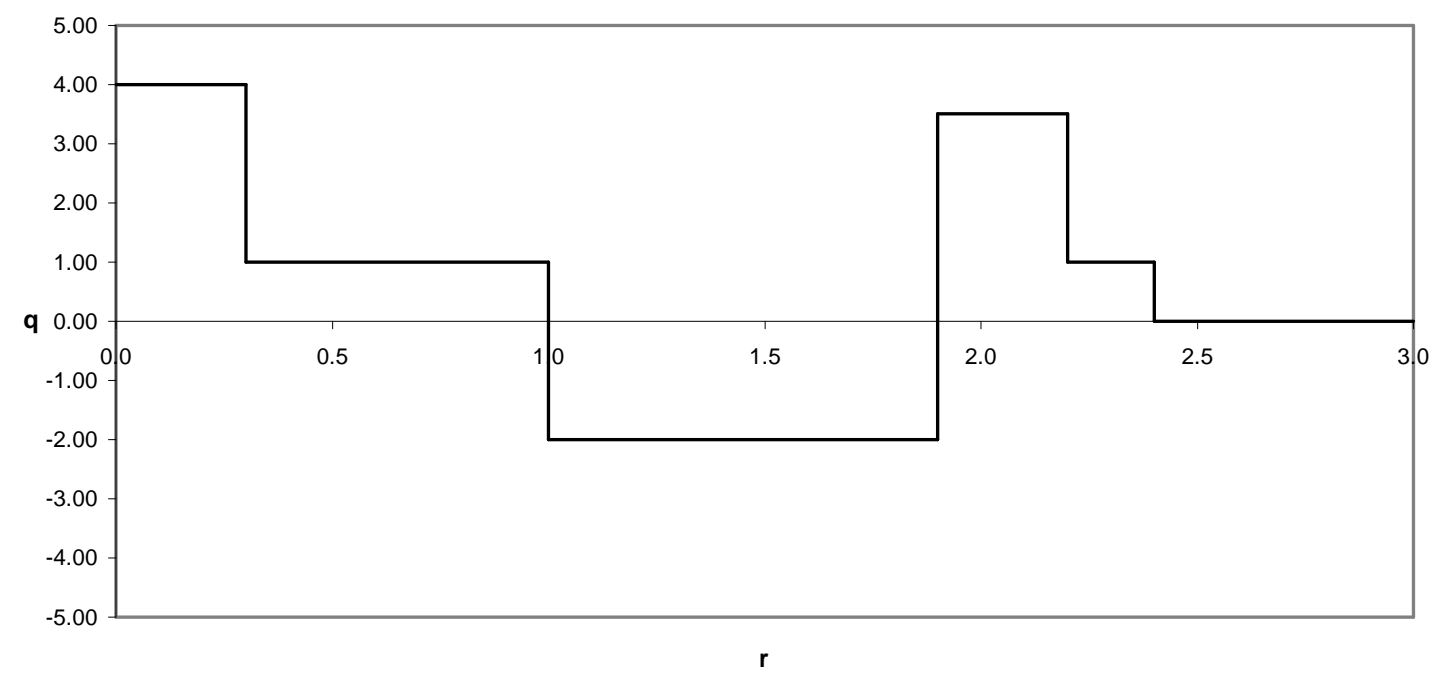

Figure 1. Potential $q_{1}(r)$.

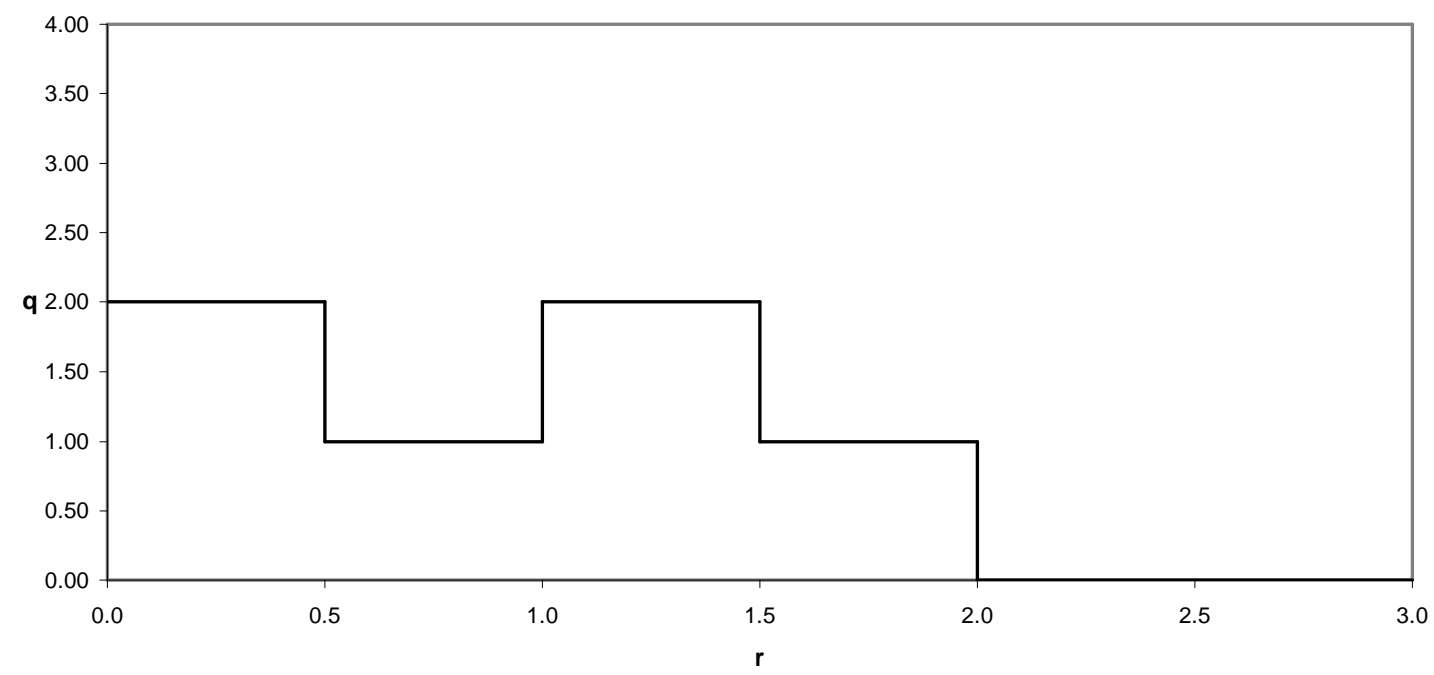

Figure 2. Potential $q_{2}(r)$. 


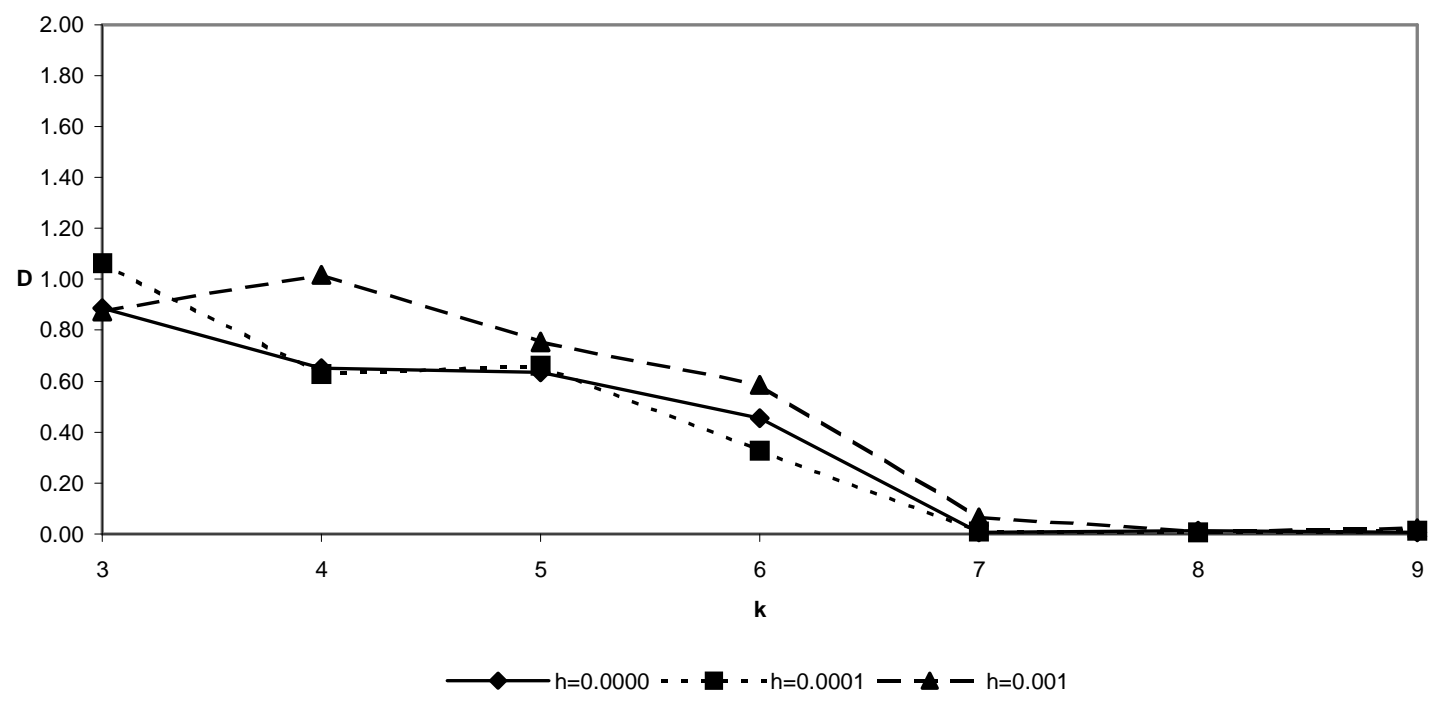

Figure 3. Diameters $D$ of minimizing sets for $q_{1}(r)$ at different noise levels $h$.

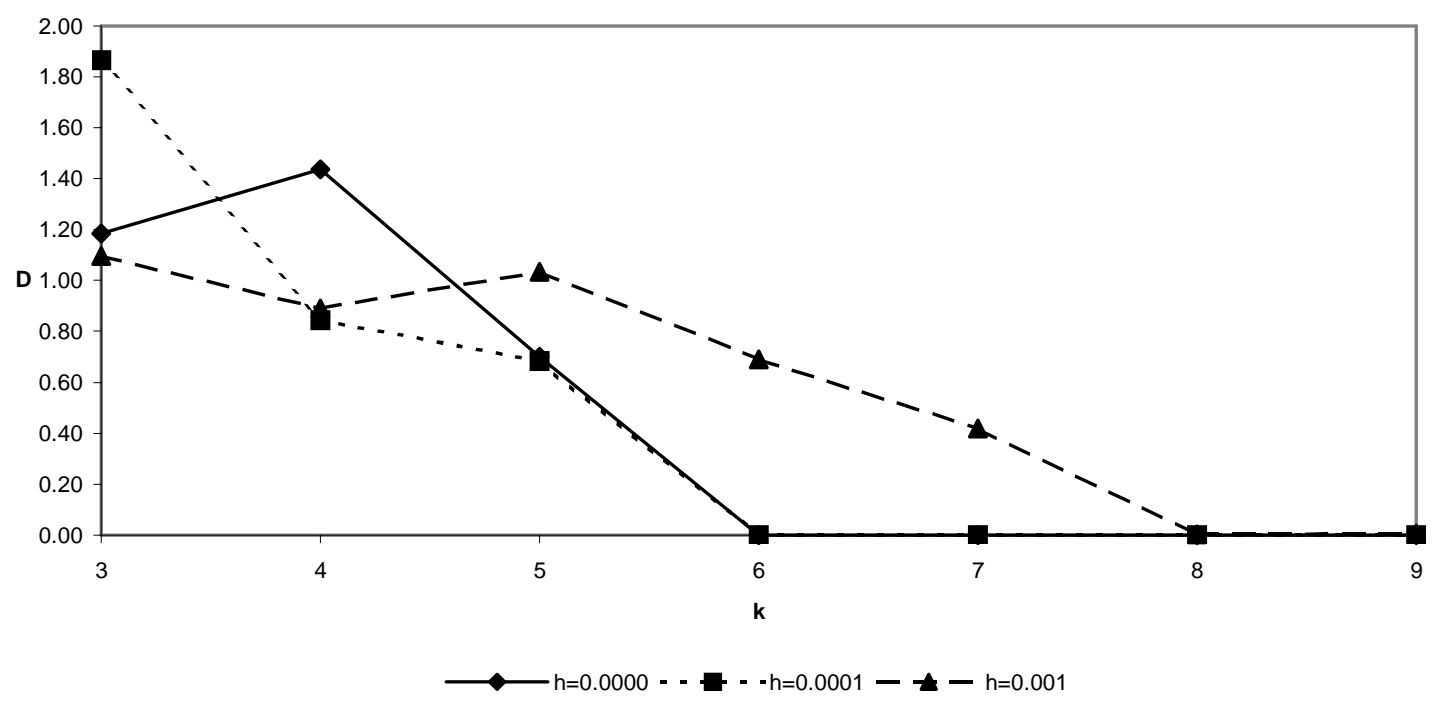

Figure 4. Diameters $D$ of minimizing sets for $q_{2}(r)$ at different noise levels $h$. 


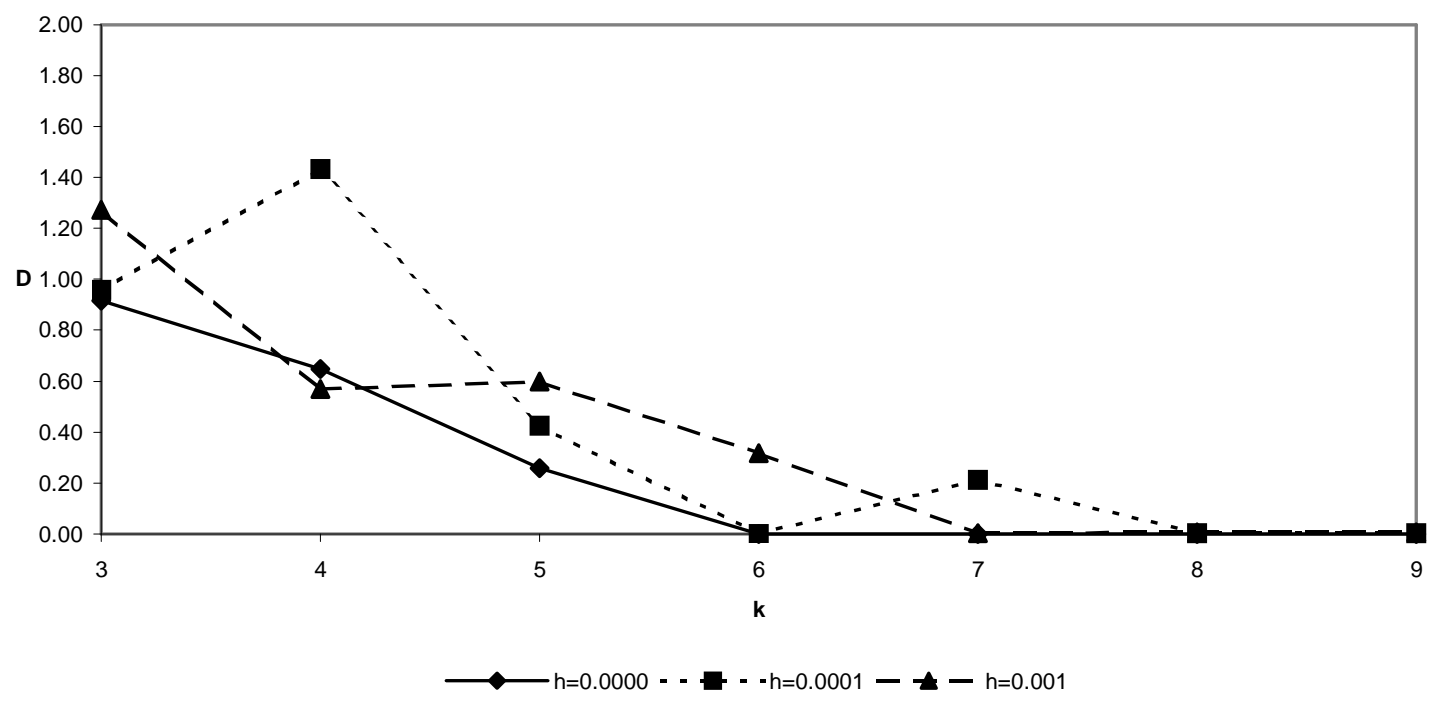

FiguRE 5. Diameters $D$ of minimizing sets for $q_{3}=0.1 q_{2}$ at different noise levels $h$.

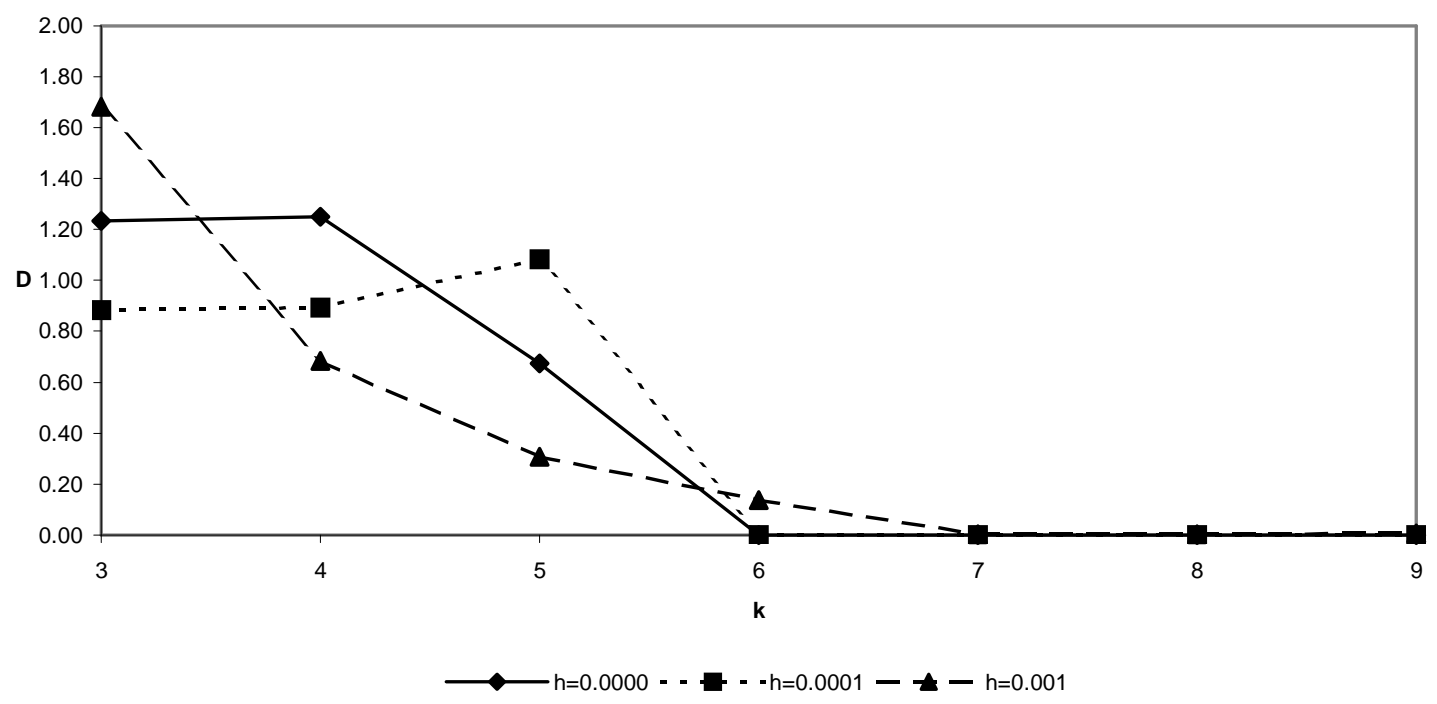

Figure 6. Diameters $D$ of minimizing sets for $q_{4}=0.01 q_{2}$ at different noise levels $h$. 